\title{
Experiências espirituais de um idoso sob cuidados paliativos: relato de caso
}

\author{
Spiritual experiences of a seriously ill elderly: a case report
}

\begin{abstract}
Alberto Gorayeb de Carvalho Ferreira ${ }^{1}$, Arthur Fernandes da Silva ${ }^{2}$, Maria Valéria Gorayeb de Carvalho ${ }^{3}$, Mirella Rebello Bezerra ${ }^{4}$
\end{abstract}

Ferreira AGC, Silva AF, Carvalho MVG, Bezerra MR. Experiências espirituais de um idoso sob cuidados paliativos: relato de caso / Spiritual experiences of a seriously ill elderly: a case report. Rev Med (São Paulo). 2015 jul.-set.;94(3):185-8.

RESUMO: Objetivo: Relatar as experiências espirituais de uma idosa internada em uma enfermaria de cuidados paliativos. Método: As informações foram estruturadas a partir de revisão de prontuário, entrevista com a paciente e acompanhante e revisão de literatura. Conclusão: O caso relatado sinaliza a importância de maior discussão sobre a atenção aos aspectos espirituais dos pacientes no fim da vida. Neste caso, por apresentar-se de forma controlada, positiva e com insight presente, entende-se o exposto como uma experiência espiritual, livre de psicopatologia. Logo, observa-se a necessidade de pesquisas futuras que objetivem o incremento da abordagem espiritual com o intuito de instrumentalizar o profissional para a sua prática.

Descritores: Espiritualidade; Cuidados paliativos; Idoso; Religião e medicina; Terapias espirituais.

\begin{abstract}
Objective: To report spiritual experiences of an elderly woman admitted to a palliative care ward. Method: Information was structured from review of medical charts, interview with the patient and her caregiver, and literature review. Conclusion: The reported case highlights the importance of having a greater discussion on the attention to spiritual aspects of patients at end of life. In this case, due to the fact of presenting herself in a controlled manner, positive and with actual insight, one should understand the exposed as a spiritual experience, free of psychopathology. Therefore, one should observe the need for future surveys aimed at enhancing the spiritual approach with the purpose of training professionals for its practice.
\end{abstract}

Keywords: Spirituality; Palliative care; Aged; Religion and medicine; Spiritual therapies.

1. Graduando de Medicina. Grupo de Estudos em Saúde e Espiritualidade (GESESP). Associação Médico-Espírita do Estado de Pernambuco (AME-EPE). Recife, Pernambuco, Brasil. E-mail: gorayeb.alberto@gmail.com.

2. Graduando de Medicina. Liga Acadêmica de Saúde e Espiritualidade (LIASE). Associação Médico-Espírita do Cariri (AME-Cariri). Barbalha, Ceará, Brasil. E-mail: tucafsilva@gmail.com.

3. Enfermeira. Grupo de Estudos em Espiritualidade e Cuidado Integral (GECI). Coordenadora do Curso de Graduação em Enfermagem da Faculdade ASCES. Caruaru, Pernambuco, Brasil. E-mail: valeriagorayeb@asces.edu.br.

4. Médica geriatra. Coordenadora do Serviço de Cuidados Paliativos do Instituto de Medicina Integral Professor Fernando FigueiraIMIP. Recife, Pernambuco, Brasil. E-mail: mirebello@outlook.com.

Casa dos Cuidados Paliativos Prof. Saulo Suassuna. Instituto de Medicina Integral Prof. Fernando Figueira (IMIP).

Endereço para correspondência: Alberto Gorayeb de Carvalho Ferreira. Rua dos Navegantes, 972 - Boa Viagem. Recife, PE, Brasil. E-mail: gorayeb.alberto@gmail.com. 


\section{INTRODUÇÃO}

atenção aos aspectos espirituais em cuidados paliativos é apontada por alguns autores como maior indicador de boa assistência ao paciente no fim da vida ${ }^{1}$. Entendendo a espiritualidade como um amplo constructo da subjetividade humana, que pode ou não vincular-se a uma doutrina religiosa, é mister que as equipes de assistência a saúde proporcionem aos pacientes, além de adequada avaliação para o manejo da dor e dos demais sintomas, suporte biopsicossocial e espiritual.

A dimensão espiritual é fator de conforto, esperança e saúde. Na perspectiva dos cuidados paliativos, a promoção do bem-estar espiritual favorece, ainda, a dignidade e a integridade da pessoa na finitude ${ }^{2}$.

O cuidado espiritual encontra um amplo espectro de possibilidades frente aos membros da equipe multiprofissional que atua em cuidados paliativos, sendo baseado nas necessidades que emergem a partir de uma correta avaliação espiritual ${ }^{3}$. Desta, podem surgir "questões espirituais complexas", que determinam um manejo criterioso, conforme recomendação de diretrizes construídas para este fim ${ }^{4}$. Nesse ínterim, um número crescente de publicações tem demonstrado a alta prevalência de experiências espirituais ou de transcendência em sujeitos não portadores de transtornos psicóticos ou dissociativos, especialmente em amostras clínicas e geralmente hospitalizadas. Tais alterações de consciência tem sido relatadas e interpretadas como tendo valor pessoal e social ${ }^{5}$.

Um conhecimento mais aprofundado sobre as experiências espirituais é legítimo, pois, ao envolver eventos de natureza visionária ou transcendental, podem suscitar o equivoco com transtornos psicóticos. Ampliando a discussão sobre a temática proposta, a quarta edição do Manual Diagnóstico e Estatístico de Transtornos Mentais (DSM-IV) trouxe à baila a categoria denominada "Problemas Religiosos ou Espirituais", no sentido de orientar a prática clínica e favorecer uma correta avaliação dos eventos. Podem-se definir problemas religiosos como sendo conflitos relacionados à fé e a doutrina religiosa do indivíduo e problemas espirituais como conflitos envolvendo a relação com questões transcendentais. Experiências de quase morte (EQM) e os desconfortos advindos de alguma vivência espiritual, positiva ou negativa, podem ser enquadradas como um problema espiritual. Estes não implicam, necessariamente, em um transtorno mental, podendo ser o reflexo do modo como o sujeito adapta-se a uma nova fase da vida ou de uma experiência com efeitos potencialmente positivos ${ }^{6}$.

A enfermidade e/ou a vivência numa unidade de cuidados paliativos, bem como qualquer outra permanência em ambiente hospitalar, pode representar uma nova fase na vida dos pacientes. Levantamentos evidenciam que a ocorrência de experiências espirituais parece ser um fenômeno frequente em pacientes oncogeriátricos hospitalizados. Em um levantamento europeu, esse índice foi de $53 \%$ entre os sujeitos avaliados ${ }^{7}$.

A falta de conhecimento a respeito das experiências espirituais dos pacientes acaba por dificultar a sua identificação bem como a avaliação do seu conteúdo, fatores essenciais ao seu correto manejo pela equipe de saúde. Ademais, a possibilidade de esses eventos passarem despercebidos aumenta se o cuidador não estiver corretamente preparado para a interpretação da dimensão espiritual do paciente ${ }^{7}$.

Este relato de caso diz respeito à ocorrência de experiências espirituais em idoso internado na Casa dos Cuidados Paliativos Prof. Saulo Suassuna do Instituto de Medicina Integral Prof. Fernando Figueira (IMIP), instituição de referência na área de Cuidados Paliativos, localizado na cidade do Recife, Pernambuco. O tema foi selecionado pois, além de ser pouco abordado na literatura, gera implicações na abordagem da saúde do idoso.

\section{MÉTODO}

As informações contidas nesta pesquisa foram obtidas através dos relatos espontâneos do paciente em estudo e seu respectivo acompanhante durante internamento na Casa dos Cuidados Paliativos Prof. Saulo Suassuna e revisão de literatura. O projeto foi submetido ao Comitê de Ética em Pesquisa Envolvendo Seres Humanos do IMIP, sendo aprovado sob o CAE $\mathrm{n}^{\circ}$ 44937115.5.0000.5201.

\section{RELATO DE CASO}

G.S.L., 69 anos, sexo feminino, viúva e procedente de Recife - PE, internada em novembro de 2014 na enfermaria de cuidados paliativos do IMIP, foi avaliada em 23 de dezembro de 2014 por pesquisadores desta instituição que requeriam informações a respeito da prevalência de sintomas depressivos nos idosos institucionalizados. Foi realizada avaliação geriátrica ampla (AGA), padronização das atividades de vida diária (AVD) e avaliação do humor através da escala de depressão geriátrica de Yesavage.

Durante a realização da entrevista, a idosa relatou, espontaneamente, experenciar situações transcendentais ao longo do internamento. Dentre estas, referiu o contato com os "anjos guardiões" que acompanham os demais pacientes daquele serviço e apontou para a maior proximidade destes quando os pacientes estão tranquilos e sem sofrimento.

A idosa relatou instituir orações diárias pela manhã e a importância desta prática no manejo das dores 
e manutenção do seu bem-estar. A paciente expressou o desejo, com o reforço do acompanhante, de trazer ao serviço água fluidificada e aplicadores de passes magnéticos, procedimentos que fazem parte dos rituais religiosos que eram adeptas. Entretanto, as mesmas temiam a reação da equipe de saúde e dos demais acompanhantes ao depararem-se com estas práticas.

A equipe de pesquisadores passou a acompanhar a idosa até a sua alta hospitalar. Neste intervalo de tempo, além de sempre demonstrar satisfação com o conteúdo das suas experiências espirituais, não foram notificados na paciente déficit global de consciência, cursando com rebaixamento dos níveis de vigília, atenção e/ou sensopercepção, bem como sinais de hiper ou hipoatividade, estado confusional ou alterações em componentes cognitivos.

\section{DISCUSSÃO}

A literatura moderna tem estimulado a consciência de que as práticas e crenças espirituais e religiosas tem grande relevância para a qualidade de vida, interação social e a saúde das pessoas a medida que envelhecem. Nesse sentido, a gerotranscendência passa a ser objeto de estudo de alguns autores que utilizam esse conceito em referência a mudanças de perspectivas em relação à vida e ao self em idosos diante da finitude ${ }^{8}$.

A história da psiquiatria evidencia um cenário protagonizado por profissionais que sempre tenderam, salvo raras exceções, a considerar experiências espirituais como transtornos mentais e envolvimento religioso como um marcador de patologia ou imaturidade psicológica. Jackson e Fuklford ${ }^{9}$ demonstraram a fragilidade da psicopatologia tradicional e da nosologia psiquiátrica em fazer distinção entre experiências espirituais e sintomas psicóticos. Esses autores afirmam a possibilidade de uma gama de quadros serem classificados como sintomas psicóticos de acordo com alguns instrumentos de avaliação, enquanto tratamse claramente de experiências espirituais, benignas no sentido de que são recebidas com satisfação pelos sujeitos, pelas pessoas de seu meio social e por não implicarem em desorganização e deterioração de suas vidas.

Casos como este seriam melhor agrupados por meio de um termo neutro e ampliado, como "experiência anômala", que designa uma experiência incomum, ou que, embora seja relatada por muitas pessoas, acredita-se diferente do habitual e das explanações usualmente aceitas como realidade, a exemplo da alucinação ${ }^{9}$.

Para melhor compreender as experiências espirituais de pacientes em cuidados paliativos, alguns autores apontam para uma caracterização dos elementos destes eventos, dividindo-os entre alterações de consciência, experiências envolvendo a divindade, experiências de quase morte, visões a beira do leito etc ${ }^{7}$. O relato apresenta elementos que correspondem à esta ultima subdivisão. Por apresentar-se de forma controlada, com sentido positivo, bem como com insight presente, tende-se a correlacionar o conteúdo exposto como uma experiência espiritual, livre de sintomatologia psicopatológica. Nesse ínterim, a literatura especializada lança mão do conceito de emergência espiritual (spiritual emergence), que se refere à eclosão de uma experiência espiritual que surge sem acarretar perturbações das funções psicológicas ${ }^{10}$.

Levantamentos correlacionam a prevalência de experiências espirituais com reações profundas, como a diminuição de sintomatologia álgica e dispneia, menores índices de ansiedade e melhores enfrentamentos diante de doenças e da morte ${ }^{7}$. Evidenciou-se a importância que a idosa depositava em suas orações diárias. De fato, a oração é uma prática milenar de diversas e distintas religiões, tradicionalmente associada a bem-estar, promoção de saúde, introspecção e espiritualidade. ${ }^{11}$ A oração pode ser vista como uma forma de coping (enfrentamento) religioso $^{12,13}$. A literatura especializada define esse termo como o modo como o sujeito utiliza a fé e a ligação com o sagrado para lidar com o estresse e os problemas da vida - ressalta-se que a fé pode incluir religião, espiritualidade e/ou crenças pessoais ${ }^{14}$. Segundo Harrison et al. ${ }^{16}$, a prevalência do coping religioso em diferentes estudos e populações varia em uma média de $60 \%$ em pacientes institucionalizados.

Este relato abre a discussão sobre o papel do profissional de saúde diante das questões espirituais dos pacientes sob cuidados paliativos, reforçando a necessidade de se ampliar o cuidado espiritual como componente da assistência em saúde. A assistência espiritual enfatiza a importância dos relacionamentos ${ }^{16}$ e acaba por promover mudanças pessoais nos profissionais de saúde, que podem favorecê-los ao encontro de maior significado em sua pratica diária de trabalho ${ }^{4}$.

\section{CONCLUSÃO}

Sugere-se, portanto, a elaboração de estratégias voltadas a identificação das experiências espirituais em pacientes sob cuidados paliativos, no sentido de esclarecer a equipe multiprofissional de saúde a respeito do caráter não patológico das mesmas. Nessa perspectiva, pesquisas futuras que objetivem o incremento da abordagem espiritual, nos campos da formação e da assistência em saúde, devem ser estimuladas com o intuito de instrumentalizar o profissional para a sua prática. 


\section{REFERÊNCIAS}

1. Williams AL. Perspectives on spirituality at the end of life: a meta-summary. Palliat Support Care. 2006;4:407-17. doi: 10.1017/S1478951506060500.

2. Bertachini L, Pessini L. A importância da dimensão espiritual na prática dos cuidados paliativos. Rev Bioethikos - Centro Universitário São Camilo. 2010;4:315-23. Disponível em: http://www.saocamilo-sp.br/pdf/bioethikos/78/Art08.pdf.

3. Borneman T, Ferrell B, Puchalski CM. Evaluation of the FICA tool for spiritual assessment. J Pain Symptom Manage. 2010;40:163-73. doi: 10.1016/j.jpainsymman.2009.12.019.

4. Puchalski C, Ferrell B, Virani R, Otis-Green S, Baird P, Bull J, Chochinov H, Handzo G, Nelson-Becker H, PrincePaul M, Pugliese K, Sulmasy D. Improving the quality of spiritual care as a dimension of palliative care: the report of the consensus conference. J Palliat Med. 2009;12:885-904. doi: 10.1089/jpm.2009.0142.

5. Cardeña E, Winkelman M. Altering consciousness. A multidisciplinary perspective. History, culture, and the humanities. Santa Barbara: Praeger Publishers; 2011.

6. Lu FG, Lukoff D, Turner R. Religious or spiritual problems. In: American Psychiatric Association (APA). DSM-IV sourcebook. Washington DC: American Psychiatric Press; 1994.

7. Renz M, Schuell Mao M, Omlin A, Bueche D, Cerny T, Strasser F. Spiritual experiences of transcendence in patients with advanced cancer. Am J Hosp Pallt Care. 2015;32:78-88. doi: $10.1177 / 1049909113512201$.

8. Erikson JM, Erikson EH. Gerotranscendencia. In: Erickson
EH. O ciclo da vida completo. Porto Alegre: Artmed; 1998.

9. Jackson M, Fulford KWM. Spiritual experience and psychopathology. Philosophy Psychiatry Psychol. 1997;4:4165. Available from: http://psychsurfer.com/for_clinicians/ Religion_files/Jackson\%20and\%20Fulford\%20-\%20 Spiritual\%20Experience\%20and\%20Psychopathology.pdf.

10. Grof S, Grof C, editores. A tempestuosa busca do ser. São Paulo: Cultrix; 1990.

11. Guimarães HP, Avezum A. O impacto da espiritualidade na saúde física. Rev Psiquiatr Clin. 2007;34:88-94. http://dx.doi. org/10.1590/S0101-60832007000700012.

12. Benson H, Stark M. Medicina espiritual: o poder essencial da cura. Rio de Janeiro; Campus; 1998.

13. Benson H, Proctor W. Relaxation revolution: enhancing your personal health through the science and genetics of mind body healing. New York: Scribner; 2010.

14. Wong-McDonald A, Gorsuch RL. Surrender to god: an additional coping style? J Psychol Theol. 2000;28:149-61.

15. Puchalski CM. Honoring the sacred in medicine: Spirituality as an essential element of patient-centered care. J Med Person. 2008;6:113-7. Available from: http:/www.jmpweb.com/ resources/articolo/N11dab44d4cb85646a7e/N11dab44d4cb 85646a7e/113117puchalski.pdf.

16. Harrison MO, Koenig HG, Hays JC, Eme-Akawari AG, Pargament KI. The epidemiology of religious coping: a review of recent literature. Int Rev Psychiatr. 2001;13:86-93. DOI: 10.1080/09540260124356 